\title{
Equity Return Modeling and Prediction Using Hybrid ARIMA-GARCH Model
}

\author{
Kaiying Sun \\ ${ }^{1}$ Department of Electrical Engineering, State University of New York (SUNY) at Buffalo, Buffalo, USA \\ Correspondence: Kaiying Sun, Department of Electrical Engineering, State University of New York (SUNY) at \\ Buffalo, Buffalo, NY 14260, USA.
}

Received: April 11, 2017

Accepted: July 4, 2017

Online Published: July 9, 2017

doi:10.5430/ijfr.v8n3p154

URL: https://doi.org/10.5430/ijfr.v8n3p154

\begin{abstract}
In this paper, a hybrid ARIMA-GARCH model is proposed to model and predict the equity returns for three US benchmark indices: Dow Transportation, S\&P 500 and VIX. Equity returns are univariate time series data sets, one of the methods to predict them is using the Auto-Regressive Integrated Moving Average (ARIMA) models. Despite the fact that the ARIMA models are powerful and flexible, they are not be able to handle the volatility and nonlinearity that are present in the time series data. However, the Generalized Autoregressive Conditional Heteroscedasticity (GARCH) models are designed to capture volatility clustering behavior in time series. In this paper, we provide motivations and descriptions of the hybrid ARIMA-GARCH model. A complete data analysis procedure that involves a series of hypothesis testings and a model fitting procedure using the Akaike Information Criterion (AIC) is provided in this paper as well. Simulation results of out of sample predictions are also provided in this paper as a reference.
\end{abstract}

Keywords: equity return, prediction, ARIMA, GARCH

\section{Introduction}

Predicting future returns of equity market is of significant interest in many domains, since equity return is a key financial variable that affects decisions of consumers, businesses and financial institutions. Timely and reliable predictions of future equity returns can therefore provide valuable information to financial market participants.

A large number of works have been devoted to the development of prediction for equity indices, (Awartani and Corradi, 2005; Chenoweth and Obradovi, 1996; Jasic and Wood, 2006; and Noh, et al., 1994). One of the most widely used univariate time series models is the Auto-Regressive Integrated Moving Average (ARIMA) model (Koreisha and Fang, 1999; and Cline and Brockwell, 1985). However, the pre-assumed linear structure of the ARIMA model becomes the key constrain. Previous studies also showed that the Generalized Autoregressive Conditional Heteroscedasticity (GARCH) models could be used in time series prediction to explain the volatility clustering effect (Awartani and Corradi, 2005; Akgiray, 1989; Engle, 2002; and Garcia, et al., 2005). In recent years, more hybrid predictive models have been proposed to apply an ARIMA model to time series data while using GARCH to explain the conditional variance in various fields (Mohammadi and Su, 2010; Chen, et al., 2011; and Amin, et al., 2012). Note that in all previous works, the potential applications of a hybrid ARIMA-GARCH model to equity returns have not been explored.

In this paper, the returns of three equity benchmark indices, Dow Transportation, S\&P 500 and VIX, are modeled and predicted. With financial time series data fetched from Federal Reserve Economic Data (FRED), a hybrid ARIMA-GARCH model is introduced to the equity return prediction research. A complete data analysis and model fitting procedure is provided in this paper, as well as a set of out of sample simulation results of the proposed hybrid ARIMA-GARCH model.

\section{Hybrid ARIMA-GARCH Model}

In this section, we specify the ARIMA model, GARCH model, and the hybrid ARIMA-GARCH model which is used in this paper.

\subsection{ARIMA Model}

The ARIMA model is a generalization of an Auto-Regressive Moving Average (ARMA) model. Both of these models are fitted to time series data either to better understand the data or to predict future points in the series. 
ARIMA models are applied in some cases where data show evidence of non-stationarity, where an initial differencing step (the "integrated" part of the model) can be applied to reduce the non-stationarity. The ARIMA process generates non-stationary series that are integrated of order $D$. Such processes are often called difference-stationary or unit root processes.

A time series that can be modeled as a stationary $\operatorname{ARMA}(p, q)$ process after being differenced $D$ times is

denoted by ARIMA $(p, D, q)$. Given a time series data $X_{t}$, the $\operatorname{ARIMA}(p, D, q)$ model can be expressed as

$$
\nabla^{D} X_{t}=a_{0}+\sum_{i=1}^{p} \phi_{i} \nabla^{D} X_{t-i}+\varepsilon_{t}+\sum_{j=1}^{q} \theta_{j} \varepsilon_{t-j}
$$

where $a_{0}$ is a constant term, $\phi_{i}$ are the parameters of the autoregressive part of the model, $\theta_{i}$ are the parameters

of the moving average part, $\nabla^{D}$ denotes a $D$-th differenced time series, and $\varepsilon_{t}: \mathrm{N}\left(0, \sigma_{t}^{2}\right)$ is an uncorrelated innovation process with zero mean.

We can also rewrite (1) with lag operator notation, $L^{i} X_{t}=X_{t-i}$, as

$$
\Phi(L)(1-L)^{D} X_{t}=a_{0}+\Theta(L) \varepsilon_{t}
$$

where $\Phi(L)=1-\sum_{i=1}^{p} \phi_{i} L^{i}$ is a stable degree $p \quad$ AR lag operator, and $\Theta(L)=1+\sum_{j=1}^{q} \theta_{j} L^{j}$ is an invertible degree $q$ MA lag operator polynomial.

ARIMA models can be estimated following the Box-Jenkins approach. The Box-Jenkins method includes an iterative three-stage modeling approach: model identification, parameter estimation and model checking. Since stationary process is a necessary condition for an ARIMA model, when the observed time series present trend and non-seasonal behavior, differencing will be applied to the data series to remove the trend.

\subsection{GARCH Model}

The GARCH models are commonly employed in modeling financial time series that exhibit time-varying volatility clustering. GARCH models attempt to address volatility clustering in an innovations process. Volatility clustering occurs when an innovations process does not exhibit significant autocorrelation, but the variance of the process changes with time. If a series exhibits volatility clustering, it suggests that past variances might be predictive of the current variance.

The general GARCH $(r, s)$ model for the conditional variance of innovations $\varepsilon_{t}$ is given by

$$
\sigma_{t}^{2}=\omega_{0}+\sum_{i=1}^{s} \alpha_{i} \varepsilon_{t-i}^{2}+\sum_{j=1}^{r} \beta_{j} \sigma_{t-j}^{2}
$$

with constrains,

$$
\left\{\begin{array}{l}
\omega_{0}>0 \\
\alpha_{i} \geq 0, i=1,2, \ldots, s \\
\beta_{j} \geq 0, j=1,2, \ldots, r \\
\sum_{i=1}^{s} \alpha_{i}+\sum_{j=1}^{r} \beta_{j}<1
\end{array}\right.
$$


where $\omega_{0}$ is a constant term, $r$ is the order of the GARCH terms $\sigma^{2}$, which represents the number of lagged conditional variances. $s$ is the order of the $\mathrm{ARCH}$ terms $\varepsilon^{2}$, which represents the number of lagged innovations.

And $\alpha_{i}$ and $\beta_{j}$ are the coefficients of the $\mathrm{ARCH}$ and GARCH parameters, respectively.

\subsection{Hybrid ARIMA-GARCH Model}

We combine the ARIMA and GARCH models which are specified in 2.1 and 2.2 together, the hybrid ARIMA $(p, D, q)$-GARCH $(r, s)$ model can be specified as

$$
\nabla^{D} X_{t}=a_{0}+\sum_{i=1}^{p} \phi_{i} \nabla^{D} X_{t-i}+\varepsilon_{t}+\sum_{j=1}^{q} \theta_{j} \varepsilon_{t-j},
$$

where $\varepsilon_{t}: \mathrm{N}\left(0, \sigma_{t}^{2}\right)$, and

$$
\sigma_{t}^{2}=\omega_{0}+\sum_{i=1}^{s} \alpha_{i} \varepsilon_{t-i}^{2}+\sum_{j=1}^{r} \beta_{j} \sigma_{t-j}^{2} .
$$

In the rest of the paper, we will apply the hybrid ARIMA $(p, D, q)$ - $\operatorname{GARCH}(r, s)$ model specified in (5) and (6) to model and predict the equity returns.
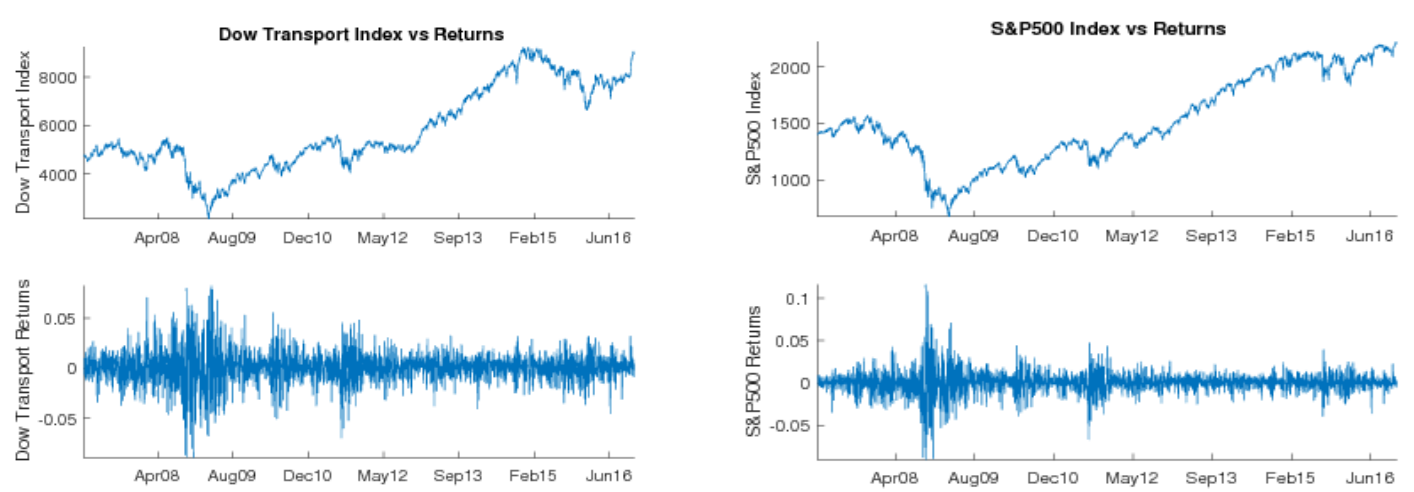

(a) Dow Transportation

(b) $S \& P 500$
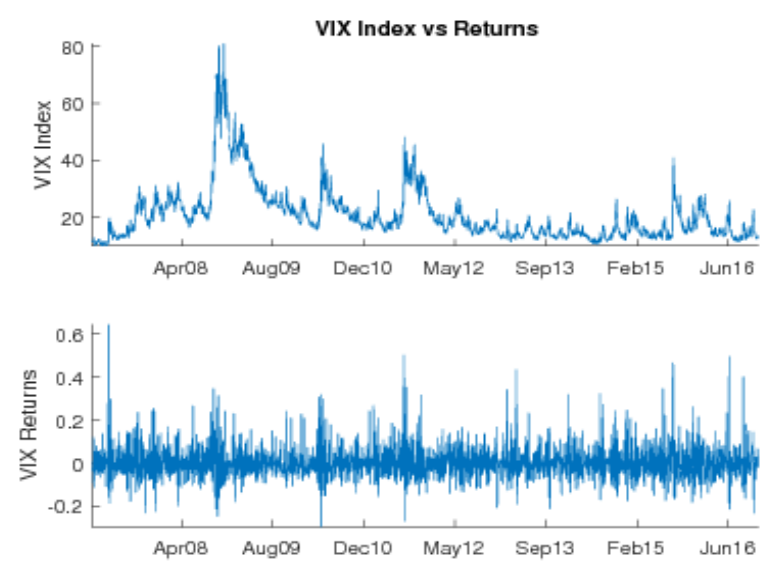

(c) VIX

Figure 1. Indices and returns performance for three benchmarks 


\section{Analysis and Modeling of Financial Data}

In this section, we provide a complete data analysis and model fitting procedure for the returns of three equity benchmark indices, Dow Transportation, S\&P 500 and VIX. Note that we analyze the historical data ranged from 12/01/2006 to 12/01/2016 for our modeling in Section 3.

\subsection{Fetching and Preprocessing of Historical Data}

We fetch the historical data from FRED with the FRED Application Programming Interface (API). Given the hybrid ARIMA-GARCH model we proposed in Section 2, we fetch the indices data for all the three benchmarks as our original financial data series. We retrieve 10 years of historical daily data of the targeted indices for a more reliable model fitting.

Since we would like to predict the daily returns of the targeted benchmark indices. We delete all missing and invalid data from the fetched series, and transform the price indices data into daily returns data series. Figure 1 shows the indices and returns performance for all the three interested benchmarks.

\subsection{Statistical Hypothesis Testings of Historical Data}

First, we test if the time series are stationary. Here we choose to apply the Phillips-Perron test since it is robust with respect to unspecified autocorrelation and heteroscedasticity in the disturbance process of the test equation compared to the Dickey Fuller test. The Phillips-Perron test let us test the existence of a unit root for a time series.

Additionally, the Kwiatkowski-Phillips-Schmidt-Shin (KPSS) test is also considered in our project, where the absence of a unit root is not a proof of stationarity but of trend-stationarity. This is an important distinction since it is possible for a time series to be non-stationary, have no unit root yet be trend-stationary. (In this project, the testing results show that the price index data of VIX is non-stationary, but trend-stationary with no unit root.) Here the KPSS test is intended to complement the Phillips-Perron test.

Besides, the Leybourne-McCabe stationarity test is applied to the time series data, which tests for the null hypothesis that the data is trend stationary, against the alternative that the data is non-stationary.

The results of the above Phillips-Perron, KPSS and Leybourne-McCabe tests show that the price indices of Dow Transportation, S\&P 500 and VIX are not stationary. However, the results of the hypothesis tests indicate that the daily returns of the three benchmark indices are stationary.

The autocorrelation and partial autocorrelation properties of the daily returns data are also studied in this project. And the corresponding results are shown in Figure 2.
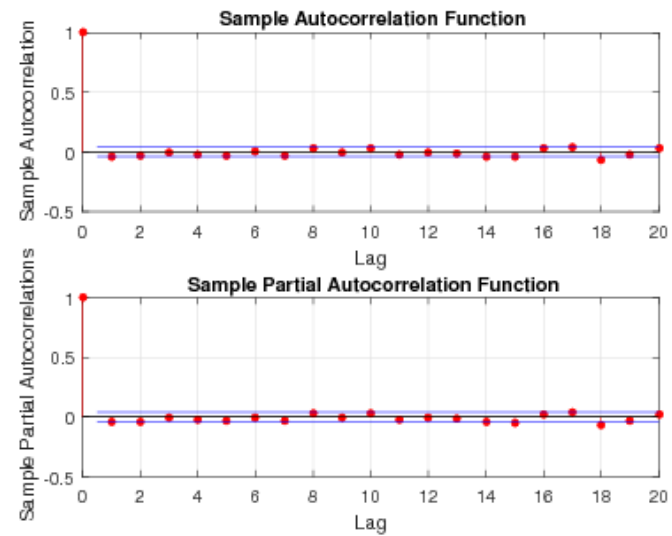

(a) Dow Transportation
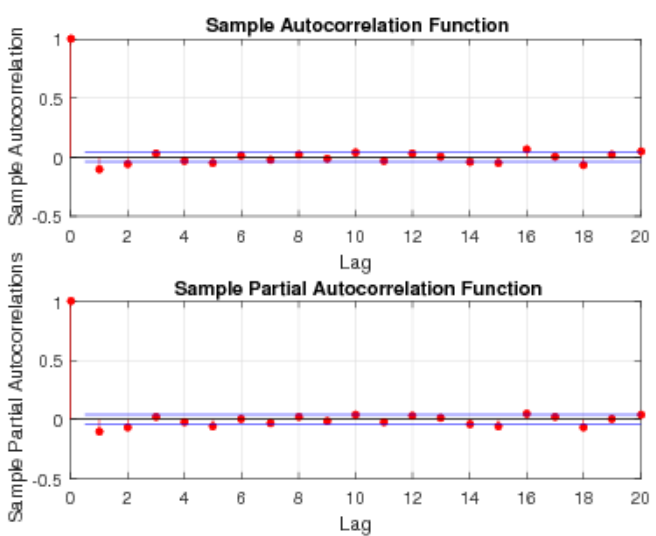

(b) S\&P 500 


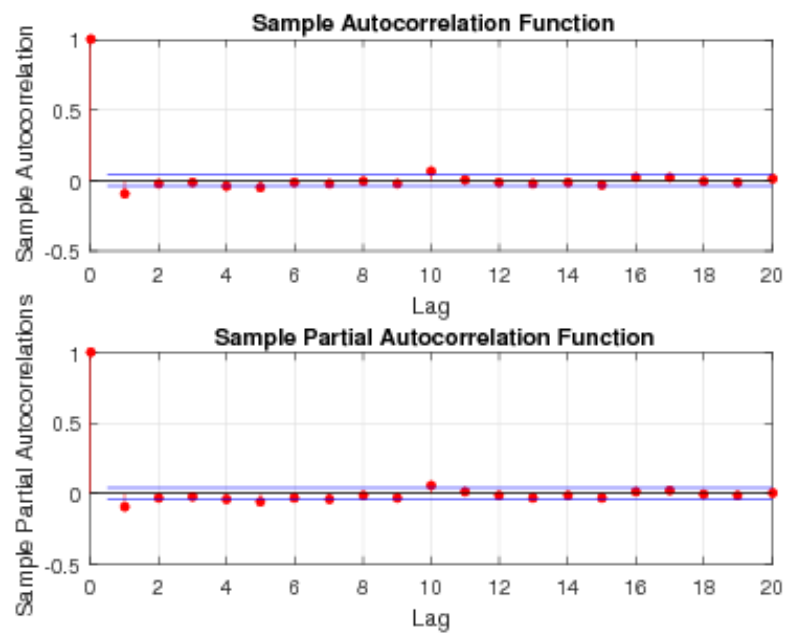

(c) VIX

Figure 2. Autocorrelations and partial autocorrelations of three benchmarks

\subsection{Lag Orders Estimation for ARIMA Model Using Akaike Information Criterion (AIC)}

In this subsection, we start to specify a model fitting procedure which estimates the orders and coefficients for the proposed hybrid ARIMA-GARCH model.

First, we estimate the lag orders for ARIMA model by comparing the Akaike Information Criterion (AIC) for different AR and MA lag orders. Although AIC does not provide a test of a model in the sense of testing a null hypothesis, it is proposed based on information theory, which offers a relative estimate of the information lost when a given model is used to represent the process that generates the data. Thus, given a collection of models for the data, AIC estimates the quality of each model, the preferred model is the one with the minimum AIC value.

In this paper, in order to find the optimal lag orders of the ARIMA model for the daily returns data of the three benchmark indices, we apply a parameter sweep by calculating the AIC values for each pair of AR and MA lags while their values vary from 1 to 8 . The optimal AR and MA lag orders for the ARIMA model are which in the pair corresponding to the minimum AIC value. Table 1 shows the optimal AR and MA lag orders for the three benchmarks.

Table 1. Optimal AR and MA Lag Orders for the return of three benchmarks

\begin{tabular}{|c|c|c|}
\hline Benchmark Indices & Optimal AR Lag Orders $p$ & Optimal MA Lag Orders $q$ \\
\hline Dow Transportation & 7 & 7 \\
\hline S\&P 500 & 1 & 2 \\
\hline VIX & 1 & 1 \\
\hline
\end{tabular}

\subsection{Testing for ARCH Effects}

Since the ARIMA model is built upon the assumption that the data is homoscedastic. In order to take into consideration the changes in variance for the returns series, we need to model the heteroscedastic behavior of the data series, which is an ARCH effect.

In this paper, we perform the Engle's ARCH test (Engle, 1982), which is a Lagrange multiplier test to assess the significance of ARCH effects, to the residuals. The residuals are calculate from the best-fitted ARIMA model in Section 3.3. The testing results indicate that ARCH effect may exist in the returns of all the three interested benchmarks. 


\subsection{Lag Orders Estimation for GARCH Model Using AIC}

In this subsection, we use the similar methodology in Section 3.3 to estimate the lag orders of GARCH lags $r$ and ARCH lags $s$ for the GARCH model. We estimate the lag orders for the GARCH model by comparing the AIC values for different pairs of GARCH and ARCH lag orders where each of them varies from 1 to 4 . The optimal lag orders correspond to the minimum AIC value. Table 2 shows the optimal GARCH and ARCH lag orders.

Table 2. Optimal GARCH and ARCH Lag Orders for the return of three benchmarks

\begin{tabular}{|c|c|c|}
\hline Benchmark Indices & Optimal GARCH Lag Orders $p$ & Optimal ARCH Lag Orders $q$ \\
\hline Dow Transportation & 2 & 2 \\
\hline S\&P 500 & 1 & 3 \\
\hline VIX & 4 & 3 \\
\hline
\end{tabular}

\subsection{Coefficients Estimation for Hybrid ARIMA-GARCH Model}

Now we can estimate the coefficients of the proposed hybrid ARIMA-GARCH model given the optimal lag orders for AR, MA, GARCH and ARCH lags found in Sections 3.3 and 3.5. The corresponding coefficients of the ARIMA and GARCH models are shown in Tables 3 and 4.

Table 3. ARIMA coefficients for the return of three benchmarks

\begin{tabular}{cccc}
\hline Benchmark Indces & Constant & AR Coefficients & MA Coefficients \\
& $a_{0}$ & {$\left[\phi_{1}, \phi_{2}, \ldots \phi_{8}\right]$} & {$\left[\theta_{1}, \theta_{2}, \ldots, \theta_{8}\right]$} \\
\hline Dow Transportation & $2.049 \times 10^{-4}$ & {$[0,0,0,0,0,0,0.679,0]$} & {$[0,0,0,0,0,0,-0.722,0]$} \\
\hline S\&P 500 & $6.741 \times 10^{-4}$ & {$[-0.075,0,0,0,0,0,0,0]$} & {$[0,-0.025,0,0,0,0,0,0]$} \\
\hline VIX & $2.568 \times 10^{-4}$ & {$[0.761,0,0,0,0,0,0,0]$} & {$[-0.851,0,0,0,0,0,0,0]$} \\
\hline
\end{tabular}

Table 4. GARCH coefficients for the return of three benchmarks

\begin{tabular}{cccc}
\hline Benchmark Indices & Constant & GARCH Coefficients & ARCH Coefficients \\
& $\omega_{0}$ & {$\left[\alpha_{1}, \alpha_{2}, \alpha_{3}, \alpha_{4}\right]$} & {$\left[\beta_{1}, \beta_{2}, \beta_{3}, \beta_{4}\right]$} \\
\hline Dow Transportation & $5.483 \times 10^{-6}$ & {$[0.866,0,0,0]$} & {$[0.017,0.092,0,0]$} \\
\hline S\&P 500 & $4.526 \times 10^{-6}$ & {$[0.808,0,0,0]$} & {$[0.049,0.11,0,0]$} \\
\hline VIX & $1.805 \times 10^{-3}$ & {$[0,0.243,0,0.193]$} & {$[0.122,0.138,0,0]$} \\
\hline
\end{tabular}

\section{Numerical Simulation for Equity Returns Using Hybrid ARIMA-GARCH Model}

In this section, we carry out some simulation results using the fitted hybrid ARIMA-GARCH model in Section 3 . In the simulation, we assume the historical data prior to date 10/01/2016 as the training data. We use the training data to estimate the model parameters and perform an out of sample prediction of 10 consecutive business days of daily 
returns from 10/03/2016 to 10/14/2016.

Given the fitted ARIMA-GARCH model, we perform Monte Carlo simulation to generate 1000 random paths, and select the median value from the simulated paths as the prediction output. Figure 3 shows the predicted returns from the simulation and the realized returns data for the three benchmark indices. Although no comparison experiments are provided to better validate the quality of the proposed modeling and predicting method, the simulation results show that the hybrid ARIMA-GARCH model is an appropriate model for predicting the equity returns.

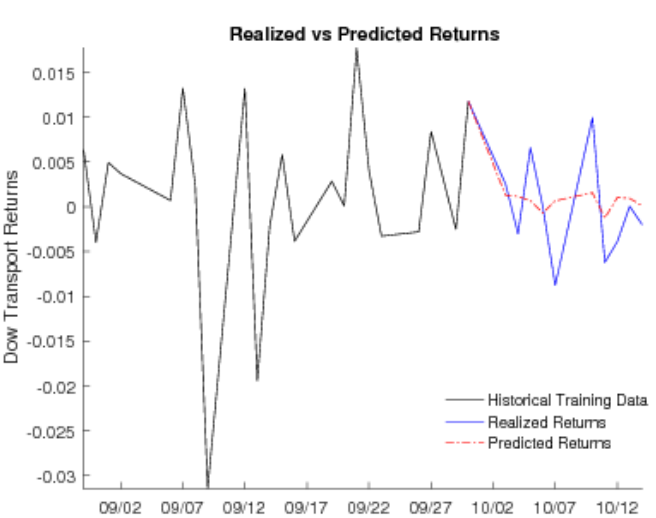

(a) Dow Transportation

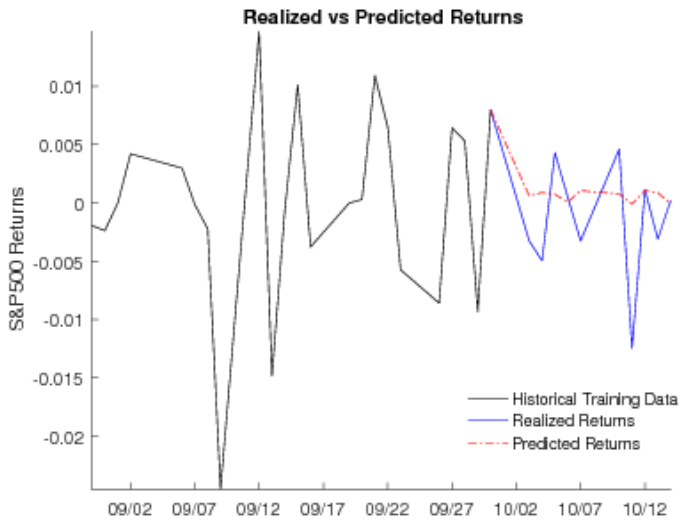

(b) S\&P 500

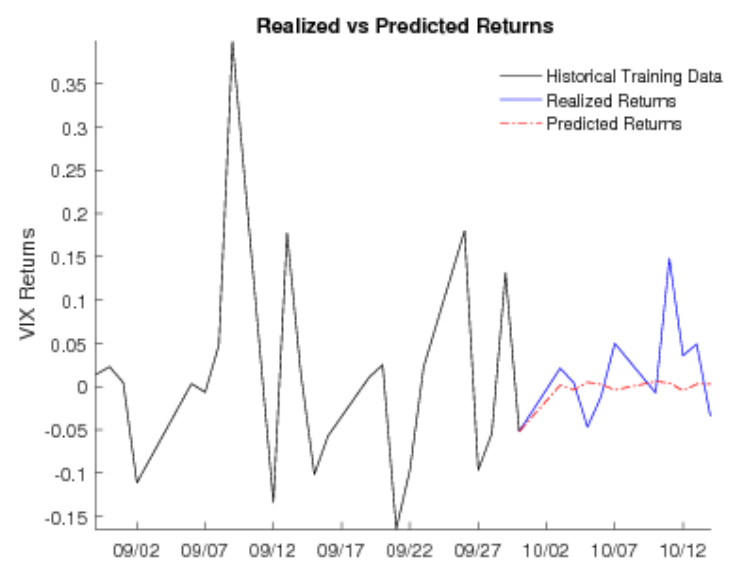

(c) VIX

Figure 3. Realized vs predicted returns performance for three benchmarks

\section{Conclusions and Future Plans}

The hybrid ARIMA-GARCH analaysis of financial equity time series data has not been explored in previous works. In this paper, we focus on modeling and prediction of the daily returns for three US benchmark indices, Dow Transportation, S\&P 500, and VIX. By defining a hybrid ARIMA-GARCH model, we analyze the returns data series and fit our model with the historical data fetched from FRED. The prediction results show that the proposed ARIMA-GARCH model is an appropriate model for predicting the equity returns.

A complete data analysis procedure that involves a series of hypothesis testings and a model fitting procedure using the AIC is provided in the project. The prediction results using the fitted ARIMA-GARCH model are also provided as a reference.

In this paper, we only specify the hybrid ARIMA-GARCH model. In the future, if we would like to further improve the prediction accuracy on equity returns, we could also try other approaches like a combination of the proposed 
ARIMA-GARCH model with the idea of artificial neural network. Previous works like (Kumar, 2009) and (Qi and Zhang, 2008) have provided some exploring trials on this topic.

\section{References}

Akgiray, V. (1989, January). Conditional Heteroscedasticity in Time Series of Stock Returns: Evidence and Forecasts. The Journal of Business, 62(1), 55-80. https://doi.org/10.1086/296451

Amin, A., Colman, A., \& Grunske, L. (2012, August). An Approach to Forecasting QoS Attributes of Web Services Based on ARIMA and GARCH Models. 2012 IEEE 19th International Conference on Web Services (ICWS).

Awartani, B. M. A., \& Corradi, V. (2005, March). Predicting the volatility of the S\&P-500 stock index via GARCH models: the role of asymmetries. International Journal of Forecasting, 21(1), 167-183. https://doi.org/10.1016/j.ijforecast.2004.08.003

Chen, C., Hu, J., Meng, Q., \& Zhang, Y. (2011, July). Short-time traffic flow prediction with ARIMA-GARCH model. 2011 IEEE Intelligent Vehicles Symposium (IV). https://doi.org/10.1109/IVS.2011.5940418

Chenoweth, T., \& Obradovi, Z. (1996, April). A multi-component nonlinear prediction system for the S\&P 500 index. Neurocomputing, 10(3), 275-290. https://doi.org/10.1016/0925-2312(95)00109-3

Cline, D. B. H., \&Brockwell, P. J. (1985, April). Linear prediction of ARMA processes with infinite variance. Stochastic Processes and their Applications, 19(2), 281-296. https://doi.org/10.1016/0304-4149(85)90030-4

Engle, R. F. (1982, July). Autoregressive Conditional Heteroskedasticity with Estimates of the Variance of United Kingdom Inflation. Econometrica, 50(4), 987-1007. https://doi.org/10.2307/1912773

Engle, R. F. (2002). New frontiers for ARCH Models. Journal of Applied Econometrics, 17, 425-446. https://doi.org/10.1002/jae.683

Garcia, R.C., Contreras, J., van Akkeren, M., \&Garcia, J. B. C. (2005, May). A GARCH forecasting model to predict day-ahead electricity prices. IEEE Transactions on Power Systems, 20(2), 867-874. https://doi.org/10.1109/TPWRS.2005.846044

Jasic, T., \& Wood, D. (2006, August). The profitability of daily stock market indices trades based on neural network predictions: case study for the S\&P 500, the DAX, the TOPIX and the FTSE in the period 1965-1999. Applied Financial Economics, 14(4), 285-297. https://doi.org/10.1080/0960310042000201228

Koreisha, S. G., \& Fang, Y. (1999). The impact of measurement errors on ARMA prediction. Journal of Forecasting, 18, 95-109. https://doi.org/10.1002/(SICI)1099-131X(199903)18:2<95::AID-FOR717>3.0.CO;2-E

Kumar, M. (2009). Nonlinear Prediction of The Standard \& Poor's 500 and The Hang Seng Index under A Dynamic Increasing Sample. Asian Academy of Management Journal of Accounting and Finance, 5(2), 101-118.

Majhi, R., Panda, G., Sahoo, G., Dash, P. K., \& Das, D. P. (2007, September). Stock market prediction of S\&P 500 and DJIA using Bacterial Foraging Optimization Technique. IEEE Congress on Evolutionary Computation.

Mohammadi, H., \& Su, L. (2010, September). International evidence on crude oil price dynamics: Applications of ARIMA-GARCH models. Energy Economics, 32(5), 1001-1008. https://doi.org/10.1016/j.eneco.2010.04.009

Noh, J., Engle, R. F., \& Kane, A. (1994). Forecasting Volatility and Option Prices of the S\&P 500 Index. The Journal of Derivatives, 2(1), 17-30. https://doi.org/10.3905/jod.1994.407901

Qim M., \& Zhang, G. P. (2008, May). Trend Time Series Modeling and Forecasting With Neural Networks. IEEE Transactions on Neural Networks, 19(5), 808-816. https://doi.org/10.1109/TNN.2007.912308 